\title{
Winkelstabile Implantate an der Brust- und Lendenwirbelsäule
}

\author{
Wolfgang Jung, Markus Schofer, Horst-Rainer Kortmann
}

\section{Zusammenfassung}

Der winkelstabile Fixateur interne in seinen verschiedenen Ausführungen stellt das Standardimplantat bei instabilen thorakolumbalen Frakturen dar Die alleinige dorsale Instrumentierung ist in der Mehrzahl dieser Verletzung ausreichend. Eine ergänzende ventrale Spondylodese bleibt ausgewählten Indikationen vorbehalten, wobei ein minimalinvasives Vorgehen diesen Indikationsbereich erweitert hat. Dies umso mehr, seitdem optimierte modulare Platten- und Stabsysteme für eine minimalinvasive Applikation entwickelt wurden. Neuere biomechanische Untersuchungen haben gezeigt, dass winkelstabile Systeme für die alleinige ventrale Spondylodese eine hohe Steifigkeit im Frakturbereich bewirken. Hieraus können für die Zukunft neue therapeutische Ansätze gewonnen werden.

\section{Einleitung}

Die dorsale Instrumentation instabiler thorakolumbaler Frakturen mit winkelstabilen transpedikulär verankerten Fixationssystemen stellt heute ein ausgereiftes Behandlungsverfahren dar $[7,14,1,20]$. Es stehen verschiedene spezielle Implantate und Instrumentarien zur Verfügung, mit denen risikoarm und schnell bei geringer Zugangsmorbidität eine Rekonstruktion der Wirbelform erzielt und eine schmerzfreie Frühmobilisation ermöglicht werden kann. Die Konstruktion des Fixateur interne mit Längsträgern, Pedikelschrauben und speziellen Backen, welche die Pedikelschrauben winkelstabil fixieren, gestattet eine wirkungsvolle dorsale Frakturreposition und Retention.

Die Entwicklung der winkelstabilen Implantate erlaubte den Schritt von der multisegmentalen zur bisegmentalen Instrumentation; in Abhängigkeit vom Frakturtyp kann die Versteifung auch monosegmental durchgeführt werden. Problematisch für den alleinig dorsalen $\mathrm{Zu}-$ gang bleibt insbesondere die dauerhaft stabile Rekonstruktion der druckbelasteten ventralen Säule.

OP-JOURNAL 2004; 20: 46-53

(c) Georg Thieme Verlag KG Stuttgart · New York
Langzeitbeobachtungen haben gezeigt, dass bei der rein dorsalen Instrumentation sekundäre Korrekturverluste von über $60 \%[13,14,15]$ resultieren können, so dass weiterhin kontrovers diskutiert wird, ob kombiniert dorsoventrale Verfahren nicht der rein dorsalen Instrumentierung überlegen sind $[7,12,20]$.

\section{Dorsale Stabilisationsverfahren - winkelstabile Implantate}

Dorsale Implantate wirken bei fehlender Tragfähigkeit der ventralen Wirbelsäule schienend im Sinne einer Abstützung oder Neutralisation. Bei nicht winkelstabilen Implantaten, wie z.B. der Kerbenplatte, und mehrsegmentaler Überbrückung wird die Dreipunktabstützung bei erhaltenem Wirbelbogen am Kyphosescheitel ausgenutzt. Ist der Bogen am Kyphosescheitel nicht erhalten, wird eine Vierpunktbiegung mit Abstützung an den Nachbarlaminae erforderlich. Wesentliche Nachteile der winkelinstabilen Implantate sind nicht nur die für eine Spondylodese erforderliche langstreckige Freilegung und Überbrückung der Fraktur, sondern vor allem auch schlechtere Repositionsund Retentionsmöglichkeiten als mit einem winkelstabilen Implantat.

Die zweite Möglichkeit einer dorsalen, schienenden Stabilisation ermöglichen neutralisierende Verfahren ohne Mehr- punktabstützung an den Wirbelbogen. Hierzu ist jedoch eine winkelstabile Verbindung zwischen Schrauben und Längsträgern obligat. Entsprechend kommen hierfür nur transpedikulär verankerte Fixationssysteme in Betracht. Den Fixateur interne gibt es mittlerweile in zahlreichen verschiedenen Ausführungen; er gilt aktuell als Standardimplantat und löst sämtliche an der Wirbelsäule erforderlichen Repositions- und Stabilisierungserfordernisse: Neutralisation, Distraktion und Zuggurtung.

Der winkelstabile Fixateur interne gilt aktuell als Standardimplantat bei instabilen theracolumbalen Frakturen.

Die in unserer Klinik benutzten Pedikelschraubensysteme des Universal Spine Systems (USS ${ }^{\circledR}$, Fa. Synthes, Abb.1 a u.b) oder des RECO ${ }^{\circledR}$-Fixateur interne (Fa. DePuy, Abb.1 cu.d) vereinen eine Drei- oder Vierpunktabstützung mit kurzstreckiger, winkelstabiler Montage. Diese Fixationssysteme ermöglichen ebenso die Korrektur von sagittalen Verschiebungen und Rotationsfehlstellungen [7,11]. Bedarfsweise kann durch die Montage von Querstabilisatoren die Stabilität der Konstruktion hinsichtlich einer Translation und Rotation erhöht werden. Wir empfehlen die Verwendung von Querträgern bei Typ-A/B-Verletzungen dann, wenn die ventrale Säule entweder traumatisch oder durch eine Dekompression stark destabilisiert worden ist, sowie generell bei allen rotationsinstabilen Typ-C-Verletzungen. Bei bisegmentalen Montagen setzen wir einen, bei mehrsegmentalen Instrumentationen zwei Querträger ein.

Winkelstabile Fixationssysteme erlauben eine schonende Operationstechnik bei gleichzeitig hoher Stabilität und Zuverlässigkeit und gestatten eine kleinere Implantatdimensionierung.

Die Erzielung der Winkelstabilität kann dabei in unterschiedlicher Weise erfolgen, z.B. nach dem Druckplattenprinzip: 
a

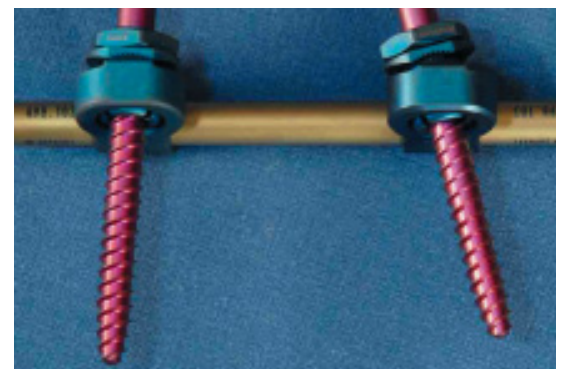

b

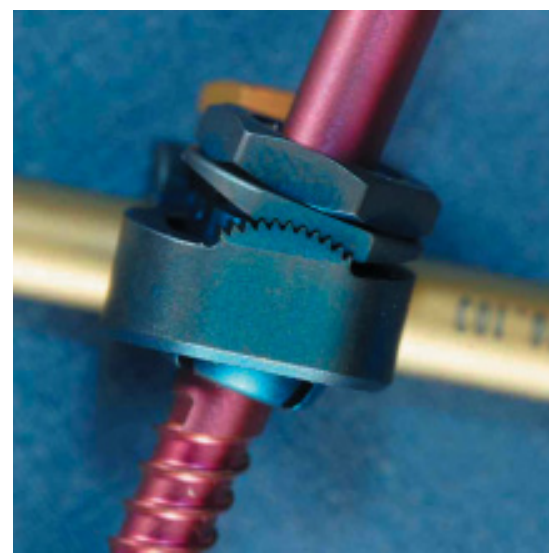

C

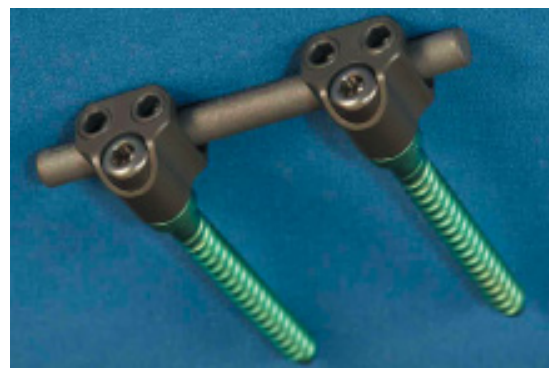

d

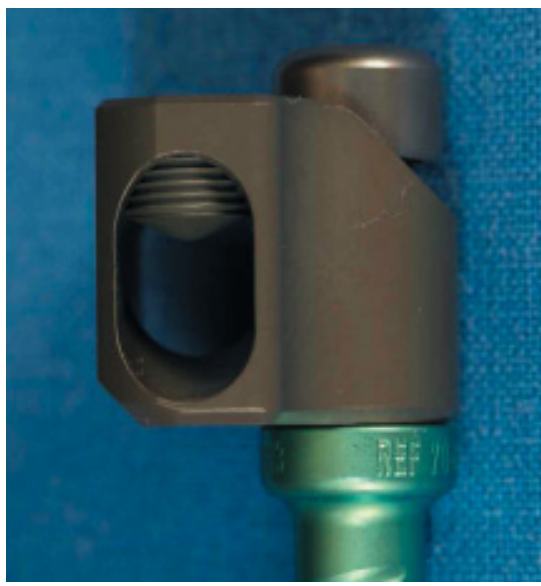

Abb.1a-d (a, b) Fixateur interne, USS ${ }^{\circledR}$; winkelstabile Fixation der Pedikelschrauben durch spezielle Backen. (c, d) Fixateur interne, Reco ${ }^{\circledR}$; winkelstabil montierte Repositionsblöcke ermöglichen eine Frakturreposition und -retention über das Implantat selbst.
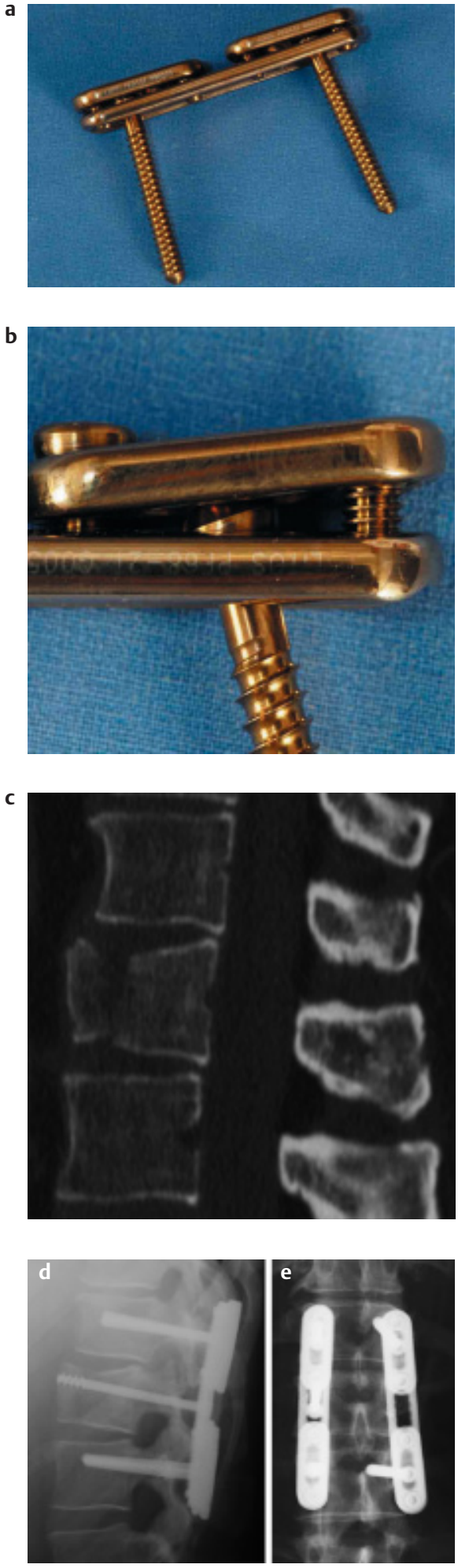

Abb.2a-e Druckplattenfixateur; multidirektionale Winkelstabilität durch Verklemmen des Schraubenkopfes im Plattenloch, „Zugschraubenosteosynthese“ bei Kneifzangenfraktur.
Bei der Verwendung des Druckplattenfixateur (Fa. Litos) wird eine multidirektionale Winkelstabilität durch das Verklemmen der Schraubenköpfe im Plattenloch durch eine aufgeschraubte Druckplatte erzielt. Mit dem Druckplattenfixateur können auch ventrale Frakturfragmente im Sinne einer „Zugschraubenosteosynthese" fixiert werden. Das Implantat erlaubt allerdings nur eine indirekte Frakturreposition (Abb.2a-d), $[16,19]$.

\section{Ventrale Stabilisierungsverfahren - winkelstabile Implantate}

Die konventionellen ventralen Zugänge zur Brust- und Lendenwirbelsäule über eine laterale Thorakotomie, Thorakophrenotomie, Lumbotomie oder Laparotomie sind standardisiert, gegenüber den dorsalen Verfahren jedoch technisch erheblich anspruchsvoller, zeitaufwändiger und vor allem komplikationsträchtiger. Hieraus folgt eine höhere Zugangsmorbidität.

Zum Aufbau der druckbelasteten ventralen Säule werden große, druckfeste Knochenspäne benötigt, die entsprechend ausgedehnte Beckenkammdefekte verursachen. Die notwendige Druckfestigkeit wird durch die Verwendung von trikortikalen Spänen erreicht. Deren hoher kortikaler Anteil macht diese Transplantate biologisch weniger wertvoll, so dass deren Integration im Sinne einer Nekrose oder Teilnekrose mit Resorption, Pseudarthrose oder Spanbruch gestört sein kann. Neuere winkelstabile Implantate erlauben eine ausschließlich ventrale Stabilisierung, wobei alternativ Vertebrektomiedefekte mit einem expandierbaren Titancage abgestützt und die knöcherne Fusion durch reine Spongiosa erreicht werden kann (Abb.3a-e).

Sekundäre Korrekturverluste konnten lange Zeit weder durch eine noch so stabile ventrale Stabilisierung noch durch ein kombiniertes dorsoventrales Vorgehen vollständig verhindert werden $[13,14,15]$, solange keine ventralen winkelstabilen Systeme zur Verfügung standen.

Die ventrale Spondylodese sollte heute mit einem winkelstabilen Implantat erfolgen. Die Winkelstabilität bewirkt eine flächenhafte Lastübertragung zwischen Knochen und Implantat über die gesamte Implantat- und Schraubenoberfläche. Die mehrpunktförmige Lastübertragung nicht winkelstabiler Implantate 

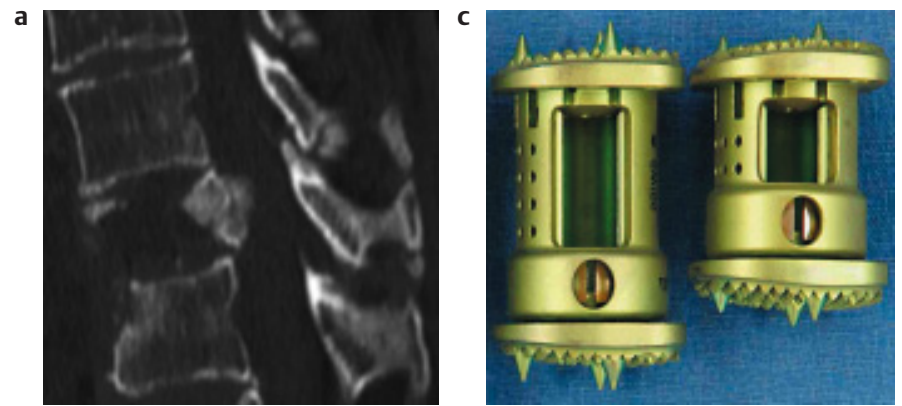

b

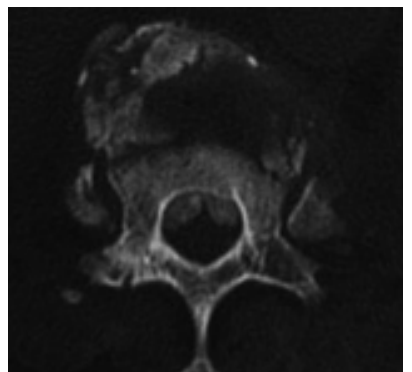

d
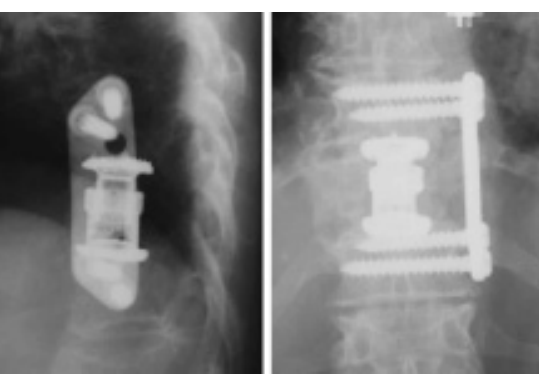

Abb.3a-e 10 Wochen alte osteoporotische BWK-10-Fraktur; Thorakotomie, Korporektomie mit Spinalkanalrevision, Implantation eines expandierbaren Synex $^{\circledR}$-Cages und ausschließlich winkelstabile ventrolaterale Plattenspondylodese (Profile ${ }^{\circledR}$ ). wird so vermieden, es resultiert eine höhere Stabilität der Osteosynthese mit reduzierter Mikrobewegung im Frakturbereich $[16,19]$.

Das Prinzip der multidirektionalen Winkelstabilität durch eine Verklemmung des Schraubenkopfes im Plattenloch findet auch bei ventralen Stabilisationssystemen im Brust- und Lendenwirbelsäulenbereich Anwendung. Basierend auf dem physikalischen Phänomen der Materialumformung kann durch die Verwendung von Titanmaterialien unterschiedlicher Härtegrade eine winkelstabile Schrauben-Platten-Verbindung im Bereich des Implantates selbst durch Hereindrängen eines gewindetragenden härteren Schraubenkofpes in ein weicheres Plattenloch realisiert werden $[16,19]$. Neuere Implantate weisen Schraubenlöcher auf, die über eine umformbare Lippe verfügen und so eine Gewindeformung überflüssig machen.

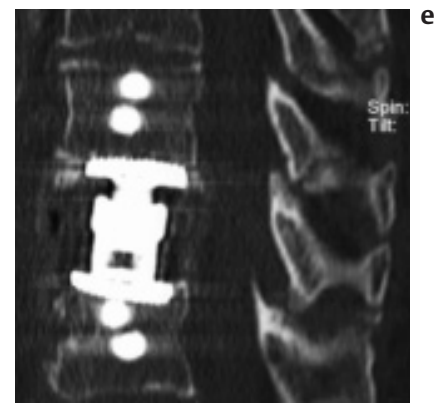

Ventrale Plattensysteme wie z.B. Profile ${ }^{\circledR}$ (Fa. DePuy) erlauben eine winkelstabile Montage durch Verwendung bikortikal verankerter Bolzen mit beidseitigem Verschlussmechanismus. Durch eine Ratschen-Zahnung können Distraktionund Kompressionsmanöver vorgenommen werden (Abb.4a-f). Andererseits wurden für die ventrale Anwendung auch winkelstabile Stabsysteme entwickelt, eine Weiterentwicklung der „locking-plate“ - Ventrofix ${ }^{\circledR}$ (Fa. Synthes) - bestehend aus einem modularen Stabsystem, wird über plattenartige Konsolen mit verriegelten Schrauben im Wirbelkörper fixiert.

Optimierte Patten- und Stabsysteme neuester Generation wie TeleFix ${ }^{\circledR}$ (Fa. Synthes, ) oder Centaur ${ }^{\circledR}$ (Fa. Stryker) sind sowohl für einen konventionellen ventralen als auch für einen minimalinvasiven Zugang konzipiert. Die modular aufgebauten Implantate können in Verbindung mit Knochentransplantaten oder kombiniert mit Wirbelkörperersatzimplantaten angewandt werden (Abb.5a-e)

\section{Indikation zum kombiniert dorsoventralen Vorgehen}

Bei der Indikationsstellung zum kombiniert dorsoventralen Vorgehen sind die Risiken des zusätzlichen ventralen Eingriffs in Relation zu der damit voraussichtlich erzielbaren Verbesserung des Behandlungsergebnisses $\mathrm{zu}$ setzen. In der Mehrzahl der instabilen thorakolumbalen Frakturen der Typen A und B ist die ausschließlich dorsale Instrumentation auch in Kenntnis eines zu erwartenden Korrekturverlustes ausreichend. Eine ergänzende ventrale Spondylodese kann einen sekundären Korrekturverlust teilweise, jedoch nicht vollständig verhindern und hat nach gegenwärtigem Kenntnisstand bei diesen Frakturtypen ansonsten auf das klinische Gesamtresultat keinen wesentlichen Einfluss. Bei den hoch instabilen Rotationsverletzungen des Typs $C$ kann durch eine alleinige dorsale Stabilisierung in der Regel jedoch keine Rotationsstabilität erreicht werden [8], so dass in diesen Fällen ein kombiniert dorsoventrales Verfahren indiziert ist.

Das ausschließlich dorsale Vorgehen hat Nachteile und Grenzen. Einerseits sind die Möglichkeiten der Spinalkanaldekompression bzw. -revision von dorsal limitiert, andererseits ist die Aufrichtung des frakturierten Wirbelsäulensegmentes durch Lordosierung und Distraktion und die Reposition von Hinterkantenfragmenten durch Ligamentotaxis umso schwieriger, unvollständiger und letztlich auch nicht mehr möglich, je später die Behandlung einsetzt. In dem Bemühen um eine dauerhaft stabile Rekonstruktion der druckbelasteten ventralen Wirbelsäulenanteile wurde inzwischen eine Indikationserweiterung zu kombiniert dorsoventralen Stabilisierungsverfahren bei allen Typ A-, B- und $C$-Läsionen diskutiert, die mit einer Verletzung der vorderen Säule im Sinne eines inkompletten oder kompletten Berstungsbruches einhergehen $[2,12]$.

Die Indikation zu kombinierten Operationsverfahren ist aus unserer Sicht in Übereinstimmung mit anderen Autoren $[1,8,9]$ zurzeit noch beschränkt auf Frakturen mit einer dorsal nicht bzw. nur unter zusätzlicher erheblicher Destabilisierung zu beseitigender Spinalkanaleinengung, auf Frakturen mit verbliebener Spinalkanaleinengung nach dorsaler Instrumentation und begleitender neurologischer Symptomatik, auf Trümmerfrakturen mit Dehiszenz nach dorsaler Instrumentierung, z.B. „Kneifzangenfrakturen“ sowie auf veraltete Frakturen. 

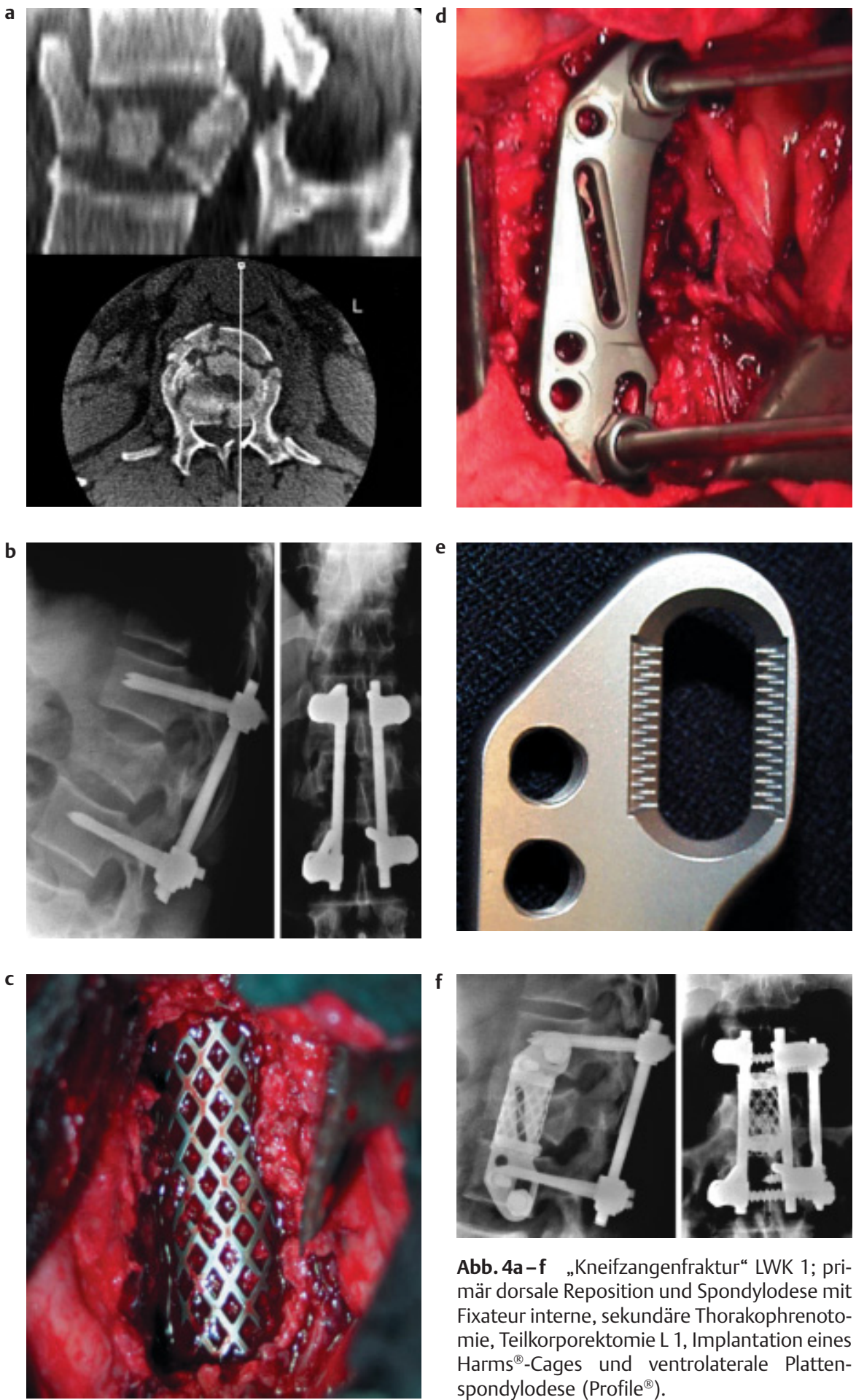

\section{Minimalinvasive Wirbelsäulenchirurgie}

In der Diskussion um den günstigsten operativen Zugang zum verletzten Wirbelsäulensegment besitzt neben biomechanischen Aspekten das Kriterium der Zugangsmorbidität einen besonderen Stellenwert.
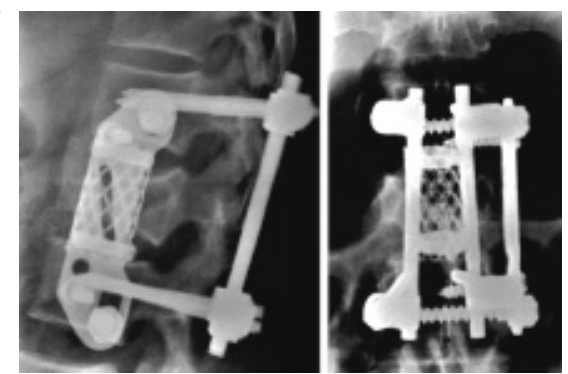

Abb.4a-f „Kneifzangenfraktur“ LWK 1; primär dorsale Reposition und Spondylodese mit Fixateur interne, sekundäre Thorakophrenotomie, Teilkorporektomie L 1, Implantation eines Harms ${ }^{\circledR}$-Cages und ventrolaterale Plattenspondylodese (Profile ${ }^{\circledR}$ ).

für eine ventrale Stabilisierung notwendige Thorakophrenikotomie vielerorts als unverhältnismäßig aufwändig angesehen und nach Möglichkeit vermieden.

Seit der Erstpublikation minimalinvasiver ventraler Spondylodesen bei Verletzungen der Brust- und Lendenwirbelsäule 1997 [6] und des endoskopischen Zwerchfellsplittings 1998 [4] wurden minimalinvasive Operationsverfahren in der Wirbelsäulenchirurgie inzwischen für nahezu den gesamten Bereich der BWS und LWS etabliert $[2,3,4,6]$ und das Indikationsspektrum für die Rekonstruktion instabiler Verletzungen der vorderen tragenden Wirbelsäulenabschnitte erweitert $[4,7,15]$.

Für die minimalinvasive Technik zur Spondylodese wurde ein speziell auf die endoskopischen Anforderungen abgestimmtes winkelstabiles Implantat (MACS TL ${ }^{\circledR}$ - modular anterior construct system for thoracic and lumbar spine, Fa. Aesculap,) entwickelt, welches die mono- und multisegmentale Stabilisierung von BWK5-LWK3 gestattet. Das modulare Implantat besteht aus einer Rahmenplatte oder alternativ einem Doppelstabsystem und ist relativ zur Hauptbelastungsrichtung der Wirbelsäule asymmetrisch aufgebaut. Das System wird durch selbstschneidende Schrauben monokortikal winkelstabil fixiert und verfügt über alle erforderlichen Repositionsmöglichkeiten. Dorsal gelegene Polyaxialschrauben sind variabel zu positionieren und realisieren ein 4-Punkt-winkelstabiles Design. Die Implantatpalette wurde zwischenzeitlich durch ein Spezialimplantat für die BWS und die MACS-TL ${ }^{\circledR}$-Polyaxial srew XL als zementierbare Verankerungsvariante bei schlechter Knochenqualität erweitert (Abb.6a-c).

Biomechanische Studien haben für dieses System eine hohe Primärstabilität im Hinblick auf Rotation, Extension und Flexion der zu überbrückenden Segmente auch bei alleinig ventraler Versorgung belegt $[3,10,17,18]$. Die Entwicklung solcher Implantate stellt einen wichtigen Schritt zu einem ausschließlich ventralen Versorgungskonzept bei instabilen Verletzungen der Typen A und B nach Magerl dar. über eine Thorakotomie ist zwar anatomisch problemlos möglich, aber im traumatologischen Patientengut durch Begleitverletzungen indikatorisch limitiert und im postoperativen Verlauf durch chronische Schmerzsyndrome belastet. Speziell für die häufigen Frakturen des thorakolumbalen Überganges wird die

Gegenwärtig lassen sich endoskopisch minimalinvasive Techniken und mikrochirurgische Techniken reduzierter Invasivität voneinander abgrenzen. 

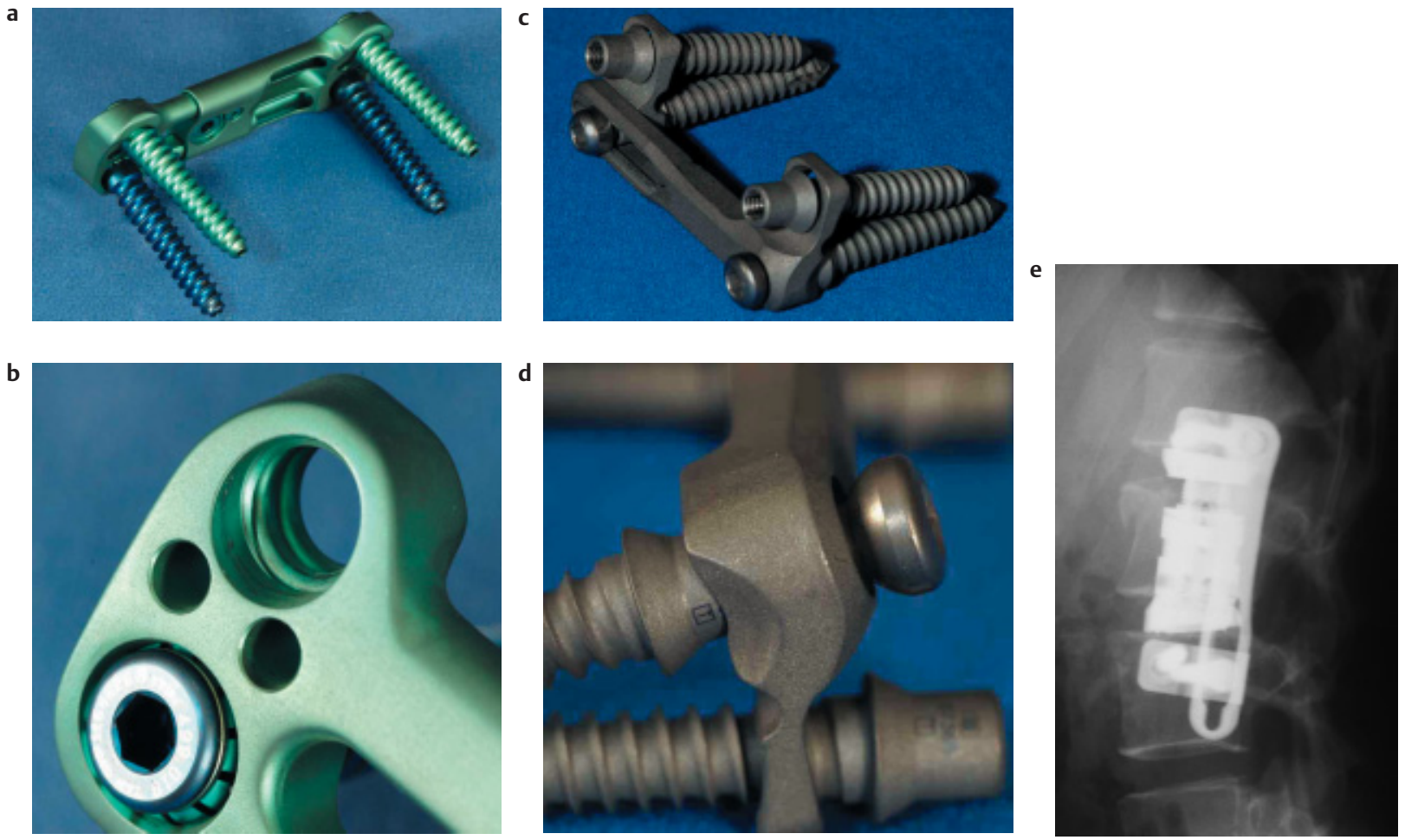

Abb.5a-e (a,b) TeleFix ${ }^{\circledR}$. (c-e) Centaur ${ }^{\circledR}$. Modulare Fixationssysteme, die eine winkelstabile Schraubenverankerung im gewindetragenden Plattenloch bzw. durch Montage einer Sicherungsschraube auf ein zylindrisches Schraubenende erzielen.

\section{Endokopische Techniken}

Die endoskopischen Operationsverfahren basieren auf videoassistierten thoraxchirurgischen Techniken, wobei die Thoraxhöhle bei seitengetrennter Beatmung über einen Doppellumentubus und ipsilateralen Lungenkollaps als vorgegebener Operationsraum dient. Unter Verwendung einer 30-Grad-Winkeloptik und überlanger Instrumente zur Weichteil- und Knochenpräparation können ventrale Spinalkanaldekompressionen, Wirbelkörperfusionen und Wirbelkörperersatzoperationen inzwischen in einem Bereich von BWK 3 bis LWK 3 vorgenommen werden. Die Exposition der thorakolumbalen Wirbelsäule gelingt durch eine endoskopische Lösung der Zwerchfellansatzes. Die Vorteile dieses Verfahren liegen in seiner Flexibilität durch die variable Platzierung des $\mathrm{Ar}$ beitstrokars im Interkostalraum und in seiner im Vergleich zur konventionellen Operation geringeren Zugangsmorbidität $[2,4,6]$.

\section{Operationsablauf}

Die Operation erfolgt oberhalb von BWK 8 aus anatomischen Gründen in Rechts-, unterhalb hiervon in Linksseitenlage und gliedert sich prinzipiell in die Schritte
Reposition, Resektion ggf. mit Spinalkanaldekompression, Knochenspanoder Cageimplantation und winkelstabiler ventrolateraler Plattenspondylodese. Die Reposition der Fraktur gelingt entweder über einen voran gehenden dorsalen Ersteingriff oder durch eine direkte instrumentelle ventrale Manipulation des betroffenen Wirbelsäulensegmentes. Die Resektion umfasst immer die verletzten Bandscheiben und verletzungsadaptiert den frakturierten Wirbelkörper im Sinne einer Korporektomie oder Teilkorporektomie.

Unter Bildwandlerkontrolle werden die zu fusionierenden Wirbel sowie die Lage des Optiktrokars und der drei Arbeitstrokare auf der Haut markiert. Nach Einbringen des Optiktrokars über eine Minithorakotomie können die weiteren Ports unter videoskopischer Kontrolle platziert werden. Durch einen fächerförmigen Retraktor kann das Lungenparenchym und ggf, das Zwerchfell beiseite gehalten werden, bei Bedarf erfolgt eine kaudale Zugangserweiterung nach Schlitzung des Zwerchfellansatzes unter Belassung eines schmalen Randsaumes. Nach Mobilisation des Musculus psoas mit dem Raspartorium und Exposition der Segmentgefäße ist der Operationssitus eingestellt.
Jeweils dorsal kranialwärts deckplattenah bzw. kaudalwärts grundplattennah im hinteren Wirbelkörperdrittel der zu instrumentierenden Wirbelkörper werden zwei kanülierte Schrauben als Plattenbasis unter Bildwandlerkontrolle eingebracht. Zwischen diesen „Landmarken“ kann anschließend die Pleura mit einem Diathermiehaken eröffnet werden. Nach Präparation und Durchtrennung der Segmentgefäße, Identifikation der Wirbelkörperhinterkante sowie der begrenzenden Bandscheiben wird mit einem Osteom die quaderförmige Ausdehnung der Wirbelkörperresektion festgelegt. Die Knochen- und Bandscheibenresektion erfolgt mit überlangen, geraden und gewinkelten Rongeuren.

Ein entsprechend zugerichteter Beckenkammspan oder expandierbarer Titancage wird nach Anfrischen der begrenzenden knöchernen Strukturen „pressfit“ in den Wirbelkörperdefekt eingepasst und ggf. durch eine aus dem frakturierten Wirbel gewonnene Spongiosaanlage ergänzt. Ein entsprechend winkelstabiles endoskopisch minimalinvasiv implantierbares Implantat sichert das Repositionsergebnis bis zur knöchernen Fusion. Nach Platzierung der Platte auf den zuvor eingebrachten dorsalen Schrauben kann die Spondylodese durch Besetzen der 

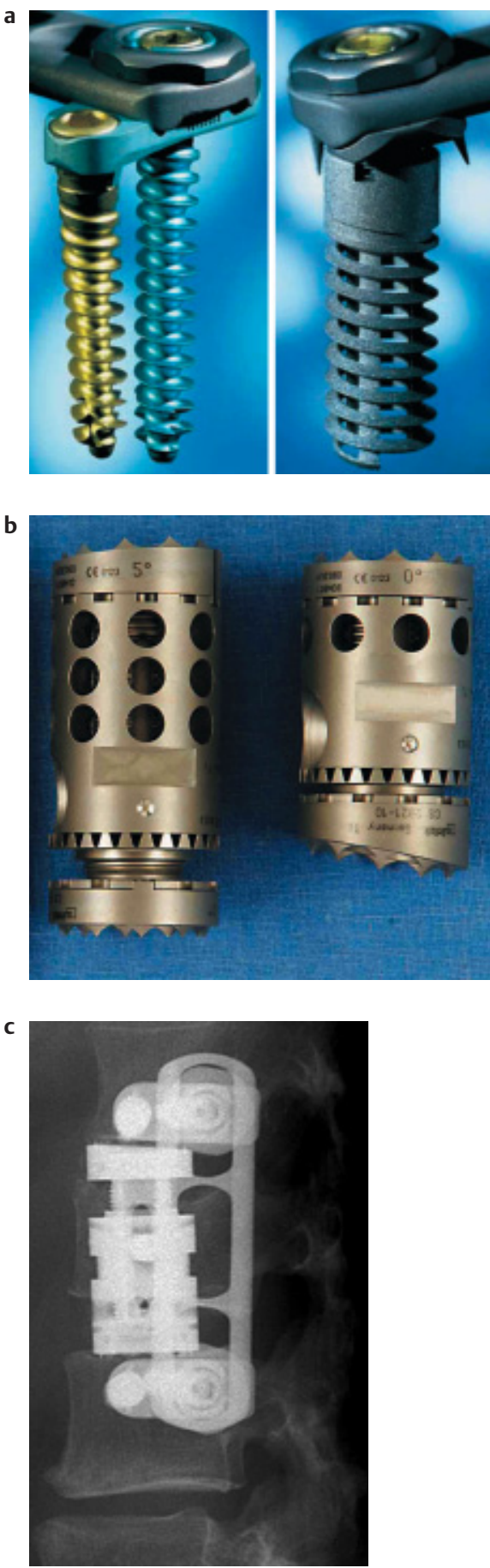

Abb.6a-c MACS-TL ${ }^{\circledR}, \quad$ MACS-TL ${ }^{\circledR}$-Polyaxialscrew-XL; modulares vollständig endoskopisch implantierbares ventrales Fixationssystem. Polyaxialität erlaubt eine freie Schraubenplatzierung und winkelstabile Implantatfixation. Minimalinvasive Spondylodese unter Verwendung eines stufenlos expandierbaren Obelisk ${ }^{\circledR}$-Cages.
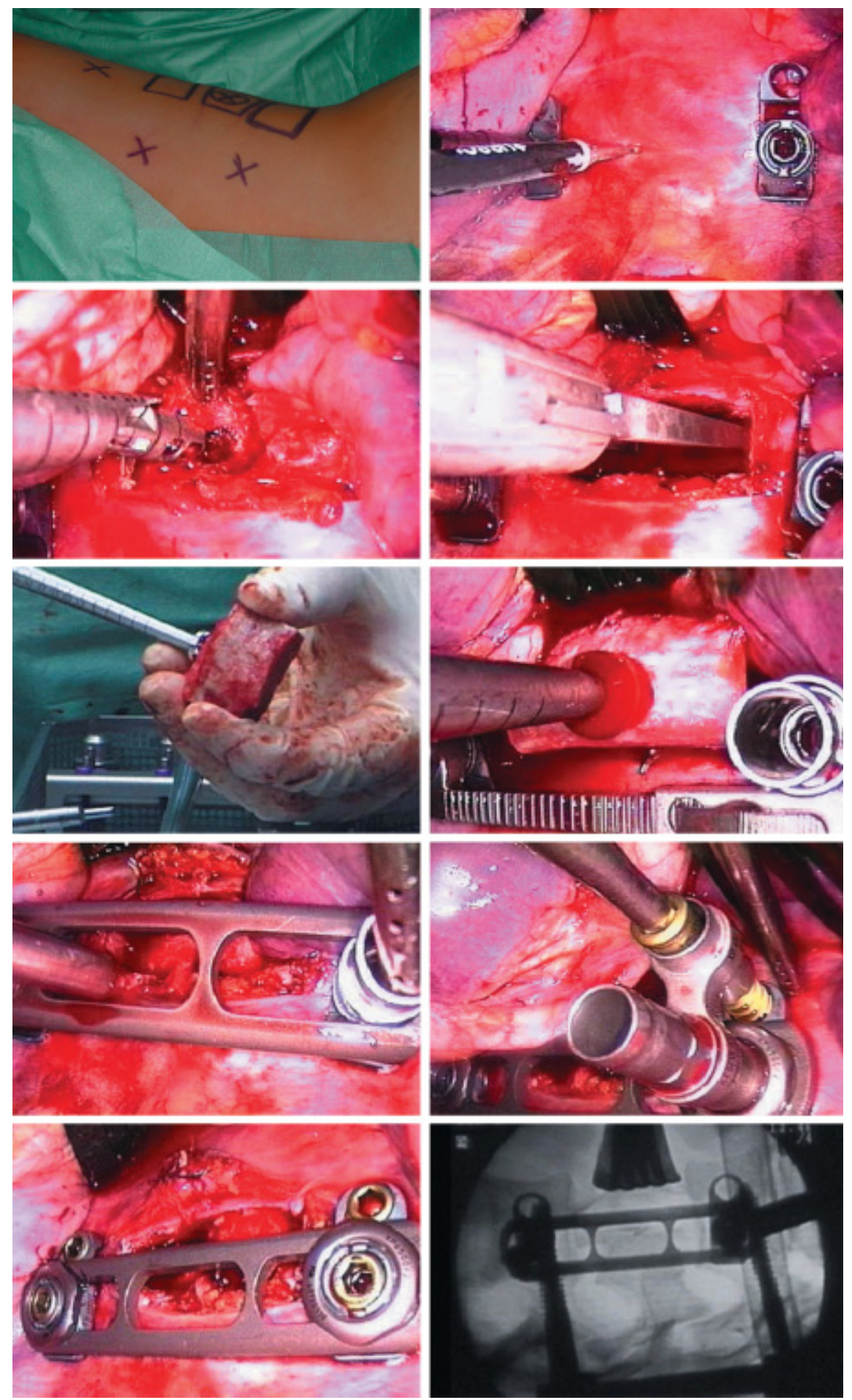

Abb. 7: Minimalinvasiver Operationsablauf; ventrolaterale Spondylodese

Th 12/L 2, endoskopisches Zwerchfellsplitting, Teilkorporektomie und Spinalkanaldekompression, Implantation eines Beckenkammspanes und winkelstabile bisegmentale Spondylodese (MACS$\left.\mathrm{TL}^{\circledR}\right)$, siehe Text. 

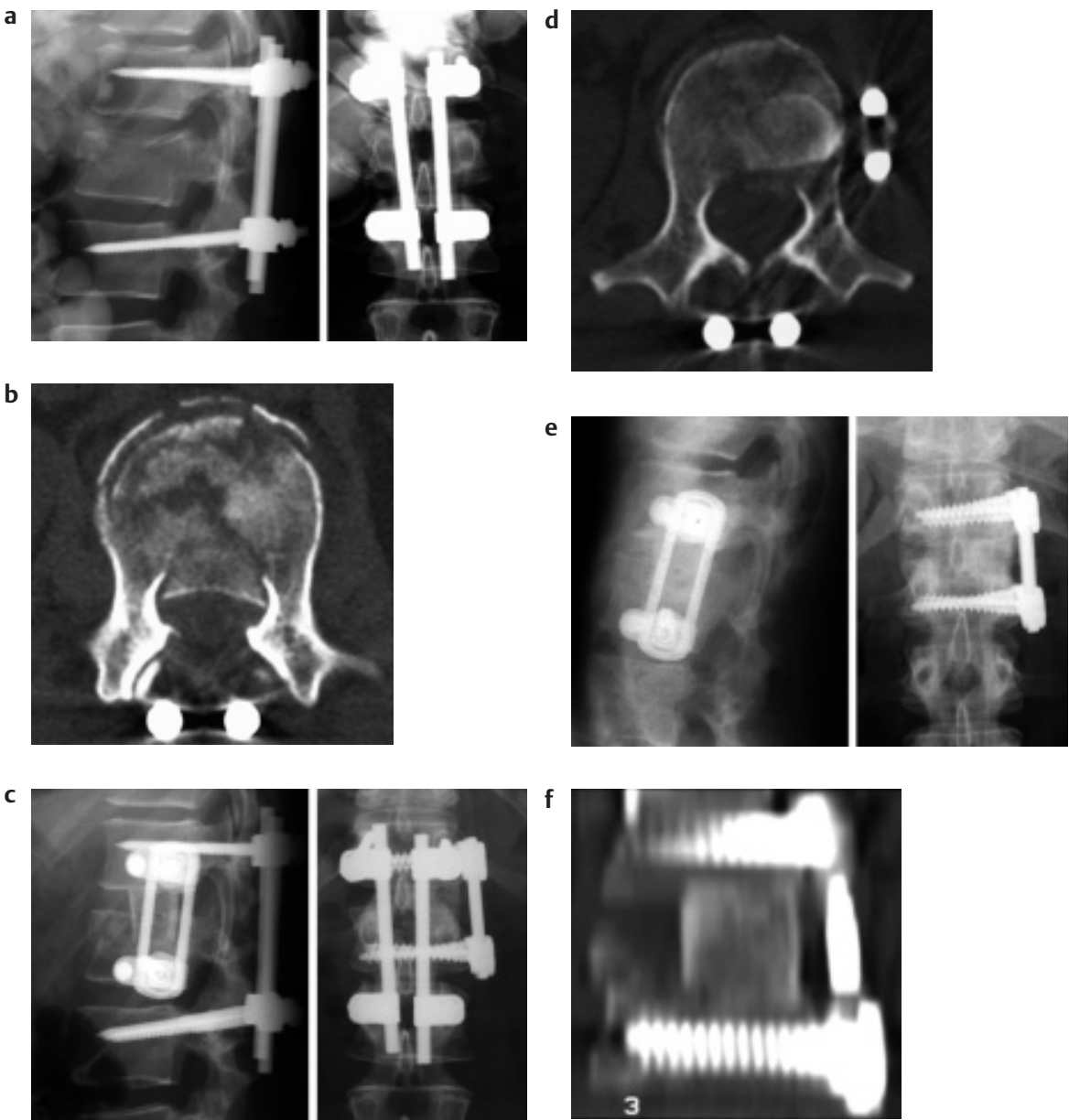

Abb.8a-f Instabile LWK1-Fraktur mit inkompletter Querschnittsymptomatik; primär dorsale Frakturreposition und Retention mit Fixateur interne (USS $\left.{ }^{\circledR}\right)$, sekundär thorakoskopisch minimalinvasive, monosegmentale ventrolaterale winkelstabile Spondylodese mit Beckenkammspan $\left(\mathrm{MACS}-\mathrm{TL}^{\circledR}\right)$.

ventralen Plattenlöcher vervollständigt werden. Mittels eines Endo-Staplers gelingt anschließend über dem Defekt der Verschluss der zuvor eröffneten Pleura [2-6], (Abb. 7).

\section{Kasuistik}

Eine 26-jährige Frau erlitt im Rahmen eines Reitunfalls eine instabile LWK1Fraktur mit deutlicher Spinalkanaleinengung und inkompletter Querschnittsymptomatik. Am Unfalltag erfolgte die Laminektomie und dorsale Instrumentierung mit einem Fixateur interne Typ USS $^{\circledR}$. Die postoperativ angefertigten konventionellen Röntgenaufnahmen zeigen eine gute Aufrichtung der Fraktur bei jedoch computertomographisch nachgewiesener fortbestehender Spinalkanaleinengung.

Am dritten postoperativen Tag konnte endoskopisch minimal invasiv die Spinalka- naldekompression mit Resektion der verletzten Bandscheibe, Teilkorporektomie sowie die monosegmentale Sprondylodese unter Verwendung eines Beckenkammspans sowie eines winkelstabilen MACS TL ${ }^{\circledR}$-Implantats durchgeführt werden. Die im Verlauf angefertigten CT-Aufnahmen zeigen eine vollständige Spinalkanaldekompression, bei radiogisch guter Frakturaufrichtung und idealer Implantat- und Transplantatlage. Bei vollständig rückläufiger neurologischer Symptomatik konnte sechs Monate später der Fixateur interne bei zu diesem Zeitpunkt belegter knöcherner Fusion des Segmentes TH 12/L 1 entfernt werden (Abb.8a-f).

\section{Schlussfolgerung}

Der winkelstabile Fixateur interne ist das Standardimplantat für die dorsale Instrumentation instabiler Frakturen des thorakolumbalen Überganges. Die ausschließ- lich dorsale Instrumentation ist in der Mehrzahl der instabilen Frakturen ausreichend. Die Indikation zu einem kombiniert dorsoventralen Vorgehen sollte vom Ausmaß der (verbliebenen) Spinalkanaleinengung und eventuellen neurologischen Symptomatik, dem Ausmaß der (verbliebenen) Instabilität und der Prognose hinsichtlich einer zeitgerechten knöchernen Konsolidierung abhängig gemacht werden. Die konventionellen ventralen Zugänge zur Brust- und Lendenwirbelsäule sind standardisiert, jedoch mit einer relativ hohen Zugangsmorbidität vergesellschaftet.

Bilanzierend ist festzustellen, dass inzwischen für die ventrale Stabilisierung winkelstabile, modulare, vollständig endoskopisch einsetzbare Implantate zur Verfügung stehen, die biomechanisch eine hohe Primärstabilität erzielen und alle Vorteile eines minimal invasiven Operationsverfahrens eröffnen. An der technischen Machbarkeit einer solchen minimal invasiven Wirbelsäulenchirurgie bestehen inzwischen keine Zweifel mehr, so dass sich parallel zu dieser technischen Entwicklung ein Trend hin zur Rekonstruktion der druckbelasteten ventralen Wirbelsäule mit entsprechender Erweiterung der Indikationsstellung abzeichnet. Ob sich minimalinvasive ventrale Operationstechniken insbesondere unter Berücksichtigung des zunehmenden Kostendruckes im „unfallchirurgischen Alltag" durchsetzen werden, bleibt abzuwarten.

\section{Literatur}

${ }^{1}$ Been HD, Bouma GJ. Comparison of two types of surgery for thoraco-lumbar fractures: combined anterior and posterior stabilisation vs. posterior instrumentation only. Acta Neurochir 1999; 141: 349-357

${ }^{2}$ Beisse R, Potulski M, Bühren V. Thorakoskopisch gesteuerte Instrumentation an BWS und LWS. Trauma Berufskrankh 2000; 2: 251-256

${ }^{3}$ Beisse R, Potulski M, Berger J, Bühren V. Entwicklung und klinischer Einsatz einer thorakoskopisch implantierbaren Rahmenplatte zur Behandlung throaco-lumbaler Frakturen und Instabilitäten. Orthopäde 2002; 31 : 413-422

${ }^{4}$ Beisse R, Potulski M, Temme C, Bühren V. Das endoskopische Zwerchfellsplitting 1998; 1001: 619-627

${ }^{5}$ Beisse R, Potulski M, Bühren V. Minimal-invasive ventrale Spondylodesen bei Verletzungen der Brust- und Lendenwirbelsäule. Chirurg 1997; 68: 1076-1084

${ }^{6}$ Bühren V. Erste Erfahrungen mit der endoskopischen Versorgung von Wirbelsäulenfrakturen mit Knochenspan und Platte. Trauma Berufskrankh 2000; 2: 19-22

7 Dick W. The „Fixateur interne“ as a versatile implant for spine surgery. Spine 1987; 12 : $882-900$ 
${ }^{8}$ Feil J, Wörsdorfer O. Ventrale Stabilisierung im Bereich der Brust- und Lendenwirbelsäule. Chirurg 1992; 63: 856-865

${ }^{9}$ Haas N, Blauth M, Tscherne H. Anterior plating in thoracolumbar spine injuries indication, technique and results. Spine 1991; 16: 100-111

${ }^{10}$ Grupp TM, Beisse R, Potulski M, Marnay T, Berger J, Blömer W. Mechanische Testung der Implantateigenschaften einer thorakoskopisch implantierbaren ventralen Wirbelsäulensabilisierungssystem. Orthopäde 2002; 31: 406-412

${ }^{11}$ Kluger P, Gerner HJ. Das mechanische Prozip des Fixateur interne zur dorsalen Stabilisierung der Brust- und Lendenwirbelsäule. Unfallchirurg 1986; 12: 68

${ }^{12}$ Knop C, Blauth M, Bastian L, Lange U, Kesting J, Tscherne H. Frakturen der thorakolumbalen Wirbelsäule. Unfallchirurg 1997; 100: 630-639

${ }^{13}$ Knop C, Blauth M, Bühren V, Hax PM, Kinzl L, Pommer A, Ulrich C, Wagner S, Weckbach A, Wentzensen A, Wörsdörfer O. Operative Behandlung von Verletzungen des thorakolumbalen Übergangs. Unfallchirurg 1999; 102: 924-935

${ }^{14}$ Knop C, Blauth M, Bühren V, Hax PM, Kinzl L, Pommer A, Ulrich C, Wagner S, Weckbach A
Wentzensen A, Wörsdörfer O. Operative Behandlung von Verletzungen des thorakolumbalen Übergangs. Unfallchirurg 2000; 103: $1032-1047$

${ }^{15}$ Knop C, Blauth M, Bühren V, Hax PM, Kinzl L, Pommer A, Ulrich C, Wagner S, Weckbach A, Wentzensen A, Wörsdörfer O. Operative Behandlung von Verletzungen des thorakolumbalen Übergangs. Unfallchirurg 2001; 104: $583-600$

${ }^{16}$ Kortmann HR. die dorsale Spondylodese bei throacolumbalen Wirbelfrakturen - Experimentalle und klinische Untersuchungen. Hefte Unfallchir 1995; 246

${ }^{17}$ Schultheiss M, Wilke HJ, Claes L, Kinzl L, Hartwig E. MACS-TL-twin-screw. Orthopäde 2002; 31: $362-367$

${ }^{18}$ Schultheiss M, Wilke HJ, Claes L, Kinzl L, Hartwig E. MACS-TL-Polyaxialscrew. Orthopäde 2002; 31: 397-401

${ }^{19}$ Seide K, Morlock M, Schürmann U, Wolter D. Wirkprinzipien der winkelstabilen PlattenSchraubenosteosynthese bei Fixateur-interne-Osteosynthesen. Trauma Berufskrankh 1999; 1: 320-325

${ }^{20}$ Tasdemiroglu E, Tibbs PA. Long-term followup results of thracolumbar fractures after posterior instrumentation. Spine 1995; 20 : $1702-1708$

\section{Dr. med. Wolfgang Jung}

Oberarzt

Dr. med. Markus Schofer

Oberarzt

Priv.-Doz. Dr. med. Horst-Rainer

Kortmann

Ärztlicher Direktor

Berufsgenossenschaftliche Unfallklinik

Duisburg GbR

Großenbaumer Allee 250

D-47249 Duisburg 